Anne Hacket

Lohnt sich Mobilität? 
Anne Hacket

\section{Lohnt sich Mobilität?}

Einkommensperspektiven

in internen und externen

Arbeitsmärkten

in den ersten Berufsjahren 
Bibliografische Information der Deutschen Nationalbibliothek

Die Deutsche Nationalbibliothek verzeichnet diese Publikation in der

Deutschen Nationalbibliografie; detaillierte bibliografische Daten sind im Internet über

$<$ http://dnb.d-nb.de> abrufbar.

\section{Auflage 2009}

Alle Rechte vorbehalten

(c) VS Verlag für Sozialwissenschaften | GWV Fachverlage GmbH, Wiesbaden 2009

Lektorat: Katrin Emmerich / Jens Ossadnik

Redaktion: Frank Seiß, ISF München

VS Verlag für Sozialwissenschaften ist Teil der Fachverlagsgruppe

Springer Science+Business Media.

www.vs-verlag.de

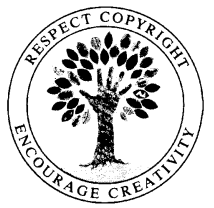

Das Werk einschließlich aller seiner Teile ist urheberrechtlich geschützt. Jede Verwertung außerhalb der engen Grenzen des Urheberrechtsgesetzes ist ohne Zustimmung des Verlags unzulässig und strafbar. Das gilt insbesondere für Vervielfältigungen, Übersetzungen, Mikroverfilmungen und die Einspeicherung und Verarbeitung in elektronischen Systemen.

Die Wiedergabe von Gebrauchsnamen, Handelsnamen, Warenbezeichnungen usw. in diesem Werk berechtigt auch ohne besondere Kennzeichnung nicht zu der Annahme, dass solche Namen im Sinne der Warenzeichen- und Markenschutz-Gesetzgebung als frei zu betrachten wären und daher von jedermann benutzt werden dürften.

Umschlaggestaltung: KünkelLopka Medienentwicklung, Heidelberg

Satz: Karla Kempgens, ISF München

Druck und buchbinderische Verarbeitung: Krips b.v., Meppel

Gedruckt auf säurefreiem und chlorfrei gebleichtem Papier

Printed in the Netherlands

ISBN 978-3-531-16300-0 


\section{Inhalt}

Vorwort und Dank einmal anders ... .......................................................... 9

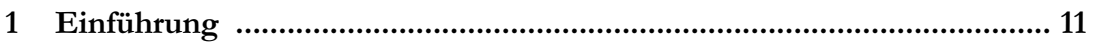

1.1 Flexibilisierung am Arbeitsmarkt - ein vieldiskutierter Begriff ................ 11

1.2 Flexibilisierung - mit welchen Folgen für die Beschäftigten? .................... 14

1.3 Die Forschungsfrage .............................................................................. 17

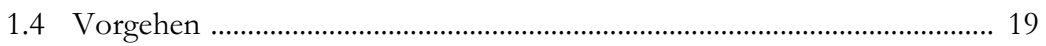

1.5 Aufbau des Bandes ...................................................................................... 21

2 Zum Forschungsstand: Betriebsstabilität, Betriebsmobilität, Einkommenseffekte ....................................................................... 23

2.1 Beschäftigungsstabilität und -instabilität ....................................................... 24

2.2 Die Erträge von Betriebsmobilität und Betriebsstabilität .......................... 43

2.3 Zusammenfassung ................................................................................ 53

3 Theorieansätze zur Erklärung der Einkommensfolgen

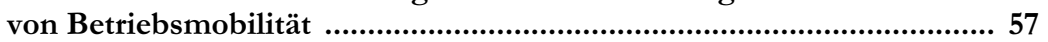

3.1 Übersicht über verschiedene theoretische Ansätze ..................................... 57

3.2 Voraussetzungen, Prognosen und ,blinde Flecken“ der Theorieansätze .. 71

3.3 Zusammenfassung und Präzisierung der Forschungsfrage ........................ 90

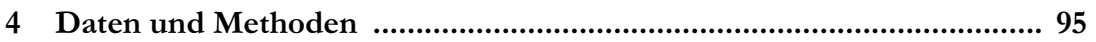

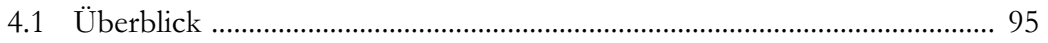

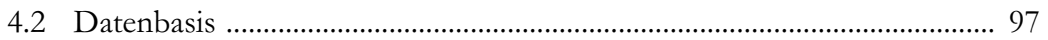

4.3 Operationalisierung der Einkommensmobilität ........................................ 104

4.4 Die Operationalisierung der Mobilität …………………………………..... 118

4.5 Die Konstruktion der Dauervariablen bis zu einem Ereignis ................. 122

4.6 Operationalisierung weiterer Variablen ....................................................... 123

4.7 Vor- und Nachteile des Untersuchungsdesigns ........................................ 124

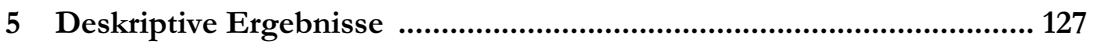

5.1 Betriebsmobilität und Betriebsstabilität ...................................................... 127

5.2 Die Wirkungen von Betriebsmobilität und -stabilität auf das Einkommen 


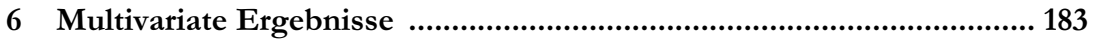

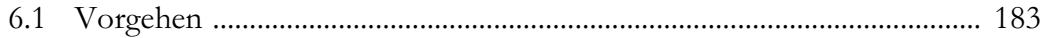

6.2 Einkommenschancen und -risiken in Ost- und Westdeutschland ......... 187

6.3 Der Einfluss von Mobilität und Stabilität auf die Einkommenschancen und -risiken ................................................................................ 189

6.4 Ergebnisse für Westdeutschland .............................................................. 192

6.5 Ergebnisse für Ostdeutschland ............................................................. 212

$7 \quad$ Lohnt sich Mobilität? Ein Fazit ............................................................. 229

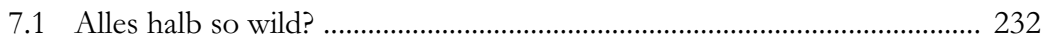

7.2 Hauptsache betriebsstabil? ........................................................................ 239

7.3 Hauptsache Arbeit im Westen? ..................................................................... 241

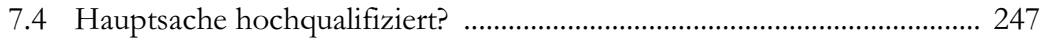

7.5 Flexicurity oder Flexploitation? ............................................................. 250

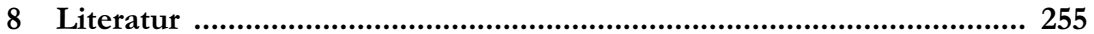

\section{Anhang A}

Die Operationalisierung der Einkommensmobilität - detaillierte

Beschreibung

\section{Anhang B}

Modell 0 - Einkommensverläufe in Ost- und Westdeutschland

Modelle aus Westdeutschland - Referenzkategorie innerregionale Mobilität (direkt) 275

Modell 1.1 - Westdeutschland - ohne Interaktionsterm ........................ 275

Modell 2.1 - Westdeutschland - mit Interaktionsterm ........................... 278

Modell 3.1 - Westdeutschland - erste Episode ........................................ 282

Modell 4.1 - Westdeutschland - zweite Episode ..................................... 285

Modell 5.1 - Westdeutschland - dritte Episode ........................................ 288

Modelle aus Ostdeutschland ............................................................................... 291

Modell 1.2 - Ostdeutschland - ohne Interaktionsterm .......................... 291

Modell 2.2 - Ostdeutschland - mit Interaktionsterm .............................. 294

Modell 3.2 - Ostdeutschland - erste Episode ………………………..... 297

Modell 4.2 - Ostdeutschland - zweite Episode ……………………….... 300

Modell 5.2 - Ostdeutschland - dritte Episode .......................................... 303 


\section{Verzeichnis der Abbildungen und Tabellen}

Abbildung 4.1: Schematische Darstellung der Operationalisierung von Einkommensereignissen ........... 113

Abbildung 4.2: Exemplarische Erwerbsverläufe innerhalb einer Einkommensepisode ............................ 115

Tabelle 4.1: $\quad$ Altersgrenzen zur Operationalisierung der Berufsanfänger je nach Qualifikation ............ 101

Tabelle 4.2: Median des Alters bei der ersten Beschäftigungsmeldung je nach Herkunft der Person und Qualifikation

Tabelle 4.3: $\quad$ Meldeort der ersten Beschäftigungsmeldung in West- und Ostdeutschland je nach Herkunft der Person ................................................................................................. 102

Tabelle 4.4: $\quad$ Überblick über die Stichprobe ......................................................................................... 103

Tabelle 5.1: $\quad$ Stabilitätsmerkmale von westdeutschen und ostdeutschen Personen ................................. 129

Tabelle 5.2: Stabilitätsmerkmale von westdeutschen und ostdeutschen Personen je nach Geschlecht

Tabelle 5.3: Wechsel des Arbeitszeitmodells bei Betriebswechseln je nach Geschlecht und je nach Herkunft der Mobilität aus Ost- und Westdeutschland

Tabelle 5.4: Wechsel des Arbeitszeitmodells bei Betriebswechsel bei Frauen je nach vorherigem Erwerbszustand und je nach Herkunft der Mobilität aus Ostund Westdeutschland

Tabelle 5.5: Stabilitätsmerkmale von westdeutschen und ostdeutschen Personen im Vergleich

Tabelle 5.6: Ziel und Richtung von Betriebswechseln je nach Herkunft der Mobilität aus Ost- und Westdeutschland

Tabelle 5.7: Mobilität je nach Herkunft der mobilen Personen sowie je nach Herkunft aus Ost- und Westdeutschland

Tabelle 5.8: Mobilität je nach Ziel und Richtung der Mobilität, je nach Herkunft der mobilen Personen sowie je nach Herkunft der Mobilität aus Ost- und Westdeutschland

Tabelle 5.9: Ziel $\quad$ Znd Richtung der Mobilität je nach Art der Mobilität und Herkunft der Mobilität aus Ost- und Westdeutschland

Tabelle 5.10: Ziel und Richtung der Mobilität je nach Qualifikation und Herkunft der Mobilität aus Ost- und Westdeutschland

Tabelle 5.11: Ziel und Richtung der Mobilität je nach Geschlecht und Herkunft der Mobilität aus Ost- und Westdeutschland

Tabelle 5.12: Anteil Zensierungen, Verluste und Gewinne je nach Episodenart .......

Tabelle 5.13: Anteil Zensierungen, Verluste und Gewinne je nach Episodenart und Episodenzahl

Tabelle 5.14: Anteil Zensierungen, Verluste und Gewinne je nach Geschlecht

Tabelle 5.15: Anteil Zensierungen, Verluste und Gewinne je nach Qualifikation

Tabelle 5.16: Einkommensgewinne und -verluste am Ende des Beobachtungszeitraums je nach Qualifikationsgruppe

Tabelle 5.17: Auswertung des Endes der jeweiligen Einkommensepisode je nach Mobilität und Ost- und Westdeutschland 
Tabelle 5.18: Auswertung des Endes der jeweiligen Einkommensepisode je nach Mobilität, Episodenzahl und Ost- und Westdeutschland

Tabelle 5.19: Anteile von zensierten Episoden und Art von Ereignis je nach Mobilität und Bildungsstand am Ende des ersten Ereignisses in Westdeutschland

Tabelle 5.20: Anteile von zensierten Episoden und Art von Ereignis je nach Mobilität und Bildungsstand am Ende des ersten Ereignisses in Ostdeutschland

Tabelle 5.21: Anteile von Zensierungen, Verlusten und Gewinnen zum Zeitpunkt des Betriebswechsels je nach Ziel und Richtung der Mobilität im Ost-West-Vergleich

Tabelle 5.22: Anteile von Zensierungen, Verlusten und Gewinnen zum Zeitpunkt einer Mobilität, differenziert nach freiwilliger und unfreiwilliger Mobilität sowie Mobilität aus einer Meldelücke

Tabelle 5.23: Einkommenseffekte von freiwilliger und unfreiwilliger Mobilität je nach Bildungsstand in Westdeutschland

Tabelle 5.24: Einkommenseffekte von freiwilliger und unfreiwilliger Mobilität je nach Bildungsstand in Ostdeutschland

Tabelle 5.25: Anteile von Zensierungen, Verlusten und Gewinnen am Ende der Einkommensepisode je nach Betriebsverbleib und indirekter Mobilität im Ost-West-Vergleich 168

Tabelle 5.26: Anteile von Zensierungen, Verlusten und Gewinnen am Ende der zweiten Einkommensepisode je nach Mobilitätsereignissen in der ersten Episode

Tabelle 5.27: Anteile von Zensierungen, Verlusten und Gewinnen am Ende der dritten Einkommensepisode je nach vorherigen Mobilitätsereignissen in Westdeutschland

Tabelle 5.28: Anteile von Zensierungen, Verlusten und Gewinnen am Ende der dritten Einkommensepisode je nach vorherigen Mobilitätsereignissen in Ostdeutschland

Tabelle 5.29: Anteile von Zensierungen, Verlusten und Gewinnen in der zweiten Episode je nach Gewinn bzw. Verlust in der ersten Episode

Tabelle 5.30: Anteile von Zensierungen, Verlusten und Gewinnen in der zweiten Episode je nach Gewinn bzw. Verlust in der ersten Episode und je nach Qualifikation

Tabelle 5.31: Anteile von Zensierungen, Verlusten und Gewinnen in der dritten Episode je nach Gewinn bzw. Verlust in der zweiten und ersten Episode

Tabelle 5.32: Die relative Einkommensposition des Anfangsgehalts je nach Mobilitätsart

Tabelle 5.33: Die relative Einkommensposition am Ende der ersten Episode je nach Mobilitätsart

Tabelle 5.34: Die relative Einkommensposition je nach Mobilität und Stabilität sowie je nach Art des Einkommensereignisses

Tabelle 6.1: Einkommensverläufe in Ost- und Westdeutschland ...

Tabelle 6.2: Einkommensverlust bei Einkommensepisoden aus Westdeutschland

Tabelle 6.3: Einkommensgewinn bei Einkommensepisoden aus Westdeutschland 196

Tabelle 6.4: Einkommensverlust bei Einkommensepisoden aus Ostdeutschland

Tabelle 6.5:

Einkommensgewinn bei Einkommensepisoden aus Ostdeutschland 217 


\section{Vorwort und Dank einmal anders ...}

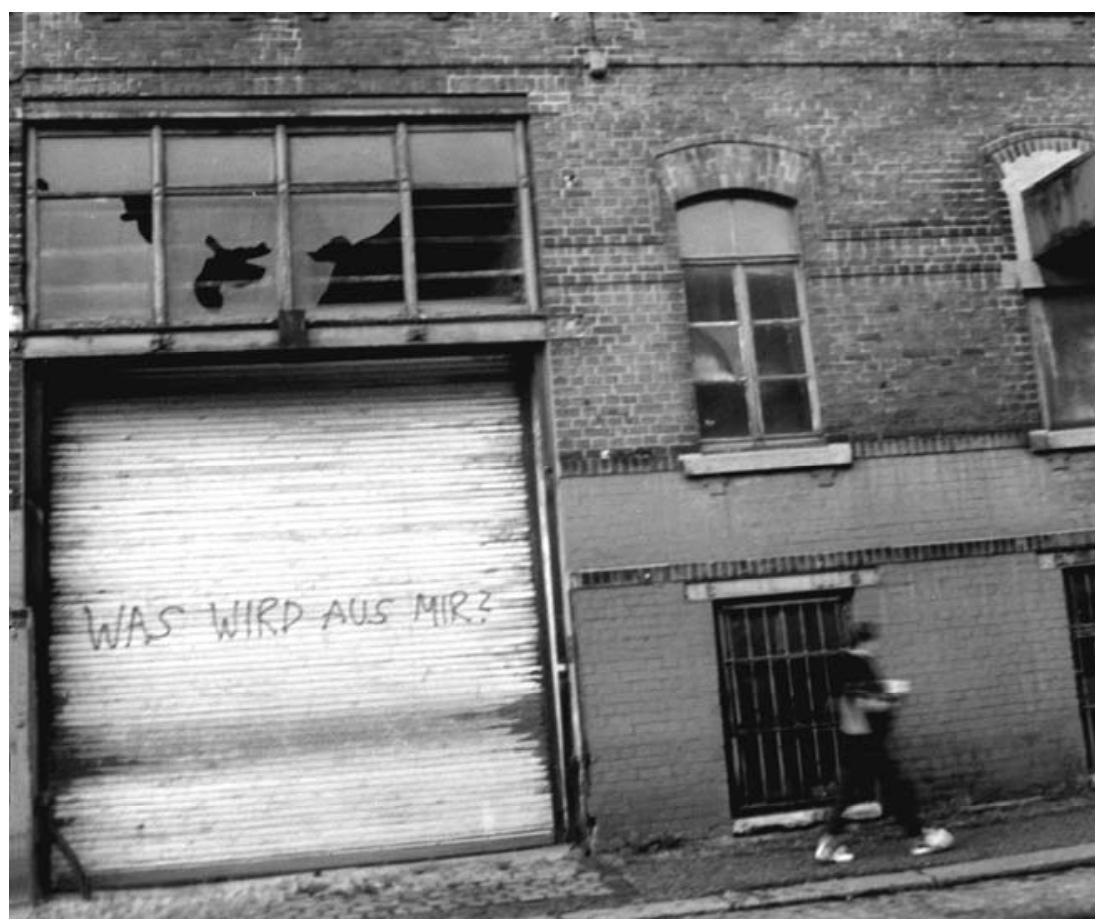

Dieses Foto entstand 1996 in Leipzig ...

Ich besuchte damals einen langjährigen Freund, der dort studierte, und war fasziniert von dem Wandel, in dem sich die Stadt Leipzig und die neuen Bundesländer befanden. Hektische Bau- und Renovierungsarbeiten, der Verfall alter Fabrikgebäude, Geschäfte und Häuser ließen deutlich spüren, dass die Entwicklung in der ehemaligen DDR mit einer starken Verunsicherung über die eigene Zukunft einherging: „WAS WIRD AUS MIR?““

Als ich etliche Jahre später mit meiner Dissertation begann und mich wissenschaftlich mit den Veränderungen des Arbeitsmarktes und den Konsequenzen für Beschäftigte unter besonderer Berücksichtigung der unterschiedlichen Situation in Ost- und Westdeutschland auseinandersetzte, kramte ich dieses Foto wieder hervor und hängte es über meinen Schreibtisch. Wenn ich mich in den komplizierten Datenaufbereitungsschritten und Operationalisierungsarbeiten verstrickte, half mir ein 
Blick auf das Bild, mich wieder meiner Ausgangsfrage zu erinnern und mich zu motivieren. So ist dieses Bild zu einem persönlichen Symbol meiner Dissertation geworden. Deswegen möchte ich es an den Anfang meiner Arbeit stellen.

Dieses Buch entstand als Dissertation im Rahmen des Projekts „Aufbereitung der Beschäftigtenstatistik der Bundesanstalt für Arbeit als Datenquelle für problemorientierte Berichtssysteme“, das von Prof. Dr. Ludwig-Mayerhofer geleitet wurde. Ihm gilt mein besonderer Dank, da er mich als Doktorvater, aber auch bereits während meiner Diplomarbeit betreut hat. Als Studentin, Diplomandin, Mitarbeiterin und Doktorandin habe ich sehr viel von ihm gelernt. Er hat es mir ermöglicht, im Rahmen eines methodisch interessanten Forschungsprojekts eigene Fragestellungen zu entwickeln und diese selbstständig zu meinem Dissertationsvorhaben zu formen. Dabei stand er mir stets mit Rat und Kritik zur Seite und war mir in methodischen und besonders in inhaltlichen Fragestellungen eine große Hilfe. Weiterer Dank gilt Prof. Dr. Rainer Geißler, der sich als Zweitgutachter dieser Arbeit angenommen hat. Ich danke auch meinen Kollegen aus dem ISF München, speziell Dr. Andreas Boes für seine motivierenden Worte, die anregenden Diskussionen sowie den Freiraum, den er mir zur Fertigstellung meiner Dissertation gewährt hat. Für die Layout- und Lektoratsarbeiten war mir die Unterstützung von Karla Kempgens und Frank Seiß sehr hilfreich.

Allen voran danke ich meiner Familie und meinen Freunden, die mich neben ihrer Unterstützung - wenn es nötig war - auch mal von der Arbeit ablenkten.

Thomas, dir danke ich für deine unermüdlichen Aufheiterungen (!), dein leckeres Essen und deine wohltuende Geduld, wenn ich ,wieder mal mitten in der Arbeit steckte“. 